\title{
AVALIAÇÃO DO COMPORTAMENTO DAS CURVAS DE APRENDIZADO DE SOLDADORES: PRODUTIVIDADE MÉDIA VERSUS PRODUTIVIDADE IDEAL
}

Versão do autor aceita publicada online: 20 set. 2021

Publicado online: 25 out. 2021

Como citar esse artigo - American Psychological Association (APA): Ferreira, M. L. R., \& Macedo, B. S. (2021). Avaliação do comportamento das curvas de aprendizado de soldadores: produtividade média versus produtividade ideal. Exacta. DOI: https://doi.org/10.5585/exactaep.2021.19995.

\section{Miguel Luiz Ribeiro Ferreira}

miguelluiz@id.uff.br

http://orcid.org/0000-0002-8576-5672

https://www.mpmontagem.uff.br/

Universidade Federal Fluminense

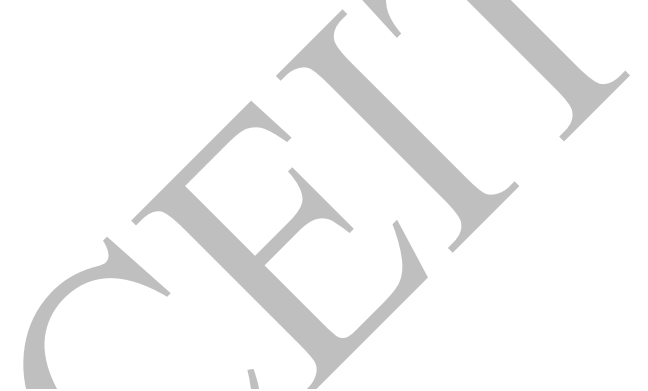

Possui graduação em Engenharia Metalúrgica pela Universidade Federal Fluminense (1979), mestrado em Engenharia Metalúrgica e de Materiais pela Universidade Federal do Rio de Janeiro (1981) e doutorado em Engenharia (Engenharia de Produção) pela Universidade de São Paulo (1998) com estágio em Cranfield University Reino Unido. Foi professor titular da Universidade Federal Fluminense, atuando no Curso de Graduação em Engenharia Mecânica, programa de pós-graduação em engenharia civil e Mestrado Profissional em Montagem Industrial. Foi Coordenador Geral dos Cursos do PROMINP da Escola de Engenharia da UFF e Coordenador do Mestrado Profissional em Montagem Industrial. Ocupou o cargo de Coordenador dos Cursos de Pós Graduação Lato Sensu da Universidade Federal Fluminense, que é um órgão vinculado a Pró Reitoria de Pesquisa, Pós Graduação e Inovação e vários cargos da administração da UFF. Desenvolveu e coordenou vários programas de treinamento e capacitação para operários, técnicos e engenheiros na área de fabricação de equipamentos e montagem industrial. Atuou por muitos anos como engenheiro na indústria de fabricação de equipamentos e montagem de instalações industriais. Desenvolveu e coordenou projetos de pesquisa na área de fabricação de equipamentos e montagem industrial. Atualmente, aposentado, atua como professor e pesquisador no Mestrado Profissional em Montagem Industrial vinculado a Escola de Engenharia da UFF, desenvolvendo projetos de pesquisa com foco em produtividade na indústria de construção e montagem de instalações industriais. Da mesma forma, atua como engenheiro prestando atividades de consultoria a empresas que atuam nos ramos de fabricação de equipamentos e montagem industrial.

\section{Bruno Sobral Macedo}

brunosobralmacedo@hotmail.com

https://orcid.org/0000-0003-0354-3695

https://www.mpmontagem.uff.br/

Universidade Federal Fluminense

Possui graduação em engenheira de produção (2011), pós graduado em engenharia de construção naval (2015) e mestre em montagem industrial (Em andamento) com experiência de 12 anos na industria naval atuando como Engenheiro de campo na construção de módulos de plataforma, Engenheiro Orçamentista elaborando orçamentos para serviços de construção, montagem e na manutenção onshore e offshore de estruturas e Engenheiro de Planejamento 
performando atividades como preparação de EAP, controle de cronogramas de construção de embarcações, histogramas para controle de mão de obra.

Resumo: Neste artigo busca-se avaliar o desempenho dos modelos de curva potencial e exponencial de aprendizagem de um grupo de soldadores, na soldagem de tubulações de aço carbono com o processo TIG. No estudo utilizou-se dois tipos de indicadores: produtividade média e produtividade ideal. A amostra agrupou os dados de produtividade, em faixas de diâmetros, de acordo com os graus de dificuldade estabelecidos pelo código ASME Seção IX. Realizou-se o ajuste dos dados aos modelos potencial e exponencial com o auxílio da ferramenta "Solver" do software Excel. Os resultados revelaram que o modelo exponencial é o que melhor representa o aprendizado. Os dados da produtividade média geraram ajustes de melhor qualidade em comparação com a produtividade ideal.

Palavras-chave: Curvas de Aprendizagem. Produtividade da Soldagem. Tubulações Industriais.

\section{BEHAVIOUR EVALUATION OF WELDERS LEARNING CURVES: AVERAGE PRODUCTIVITY VERSUS BASELINE PRODUCTIVITY}

Abstract: This article aims to evaluate the performance of the models of potential and exponential curve of learning of a group of welders, in the welding of carbon steel pipes with the TIG process. In the study, two types of indicators were used: average productivity and baseline productivity. The sample grouped productivity data, in diameter ranges, according to the degrees of difficulty established by the ASME code Section IX. Data was adjusted to the potential and exponential models with the assistance of the Excel software "Solver" tool. Results revealed that the exponential model is the one that best represents learning. Average productivity data generated better quality adjustments compared to baseline productivity..

Palavras-chave: Learning Curves. Welding Productivity. Industrial Pipes.

\section{Introdução}

A produtividade é um fator-chave para o crescimento econômico e a prosperidade em qualquer país (Vogl e Wahab, 2015; Arditi e Machtar, 2000; Kapelko, Horta, Camanho, \& Lansink, 2015) além de impactar, decisivamente, no sucesso dos projetos de construção (Islam \& Khadem, 2013; Mahamid, 2013; Loosemore, 2014; Pellegrino \& Constantino, 2018). 


\section{Referencial Teórico}

As curvas de aprendizado se baseiam no fenômeno em que a produtividade melhora através da realização de tarefas repetitivas realizadas por um trabalhador ou um grupo de trabalhadores (Anzanello, 2004; Pellegrino, Constantino, Pietroforte, \& Sancillo, 2012). Na indústria da construção os modelos potencial e exponencial são os mais usados (Anzanello, 2004; Cadaval Júnior, Duarte, \& Paes, 2013; Leite, 2002; Anzanello \& Fogliatto; 2007; Ralli, Panas, Pantouvakis, \& Karagiannakidis, 2020). Everett e Farghal (1997) e Thomas, (2009) consideram o modelo potencial o mais adequado para projetos de construção, sendo que as curvas elaboradas a partir de dados unitários apresentam melhores resultados, principalmente, nas fases iniciais da obra.

Lee, Lee, e Park (2015) em artigo sobre a aplicabilidade das curvas de aprendizagem na construção de "arranha-céus" na Coreia do Sul, mencionam as dificuldades de aplicação das curvas de aprendizagem. Os autores consideram o modelo potencial o mais utilizado e propõem sua modificação com a introdução de três fatores: mudança e adaptação das tarefas, adaptação às condições de trabalho do canteiro de obras e altura dos andares dos prédios. Hattingh, van Waveren e Chan (2019) também mencionam as dificuldades da utilização das curvas de aprendizagem em projetos de construção e realizaram um Survey, entre profissionais deste setor, concluindo que a confiabilidade da sua aplicação está associada ao atendimento de uma série de requisitos. Brockmann e Brezinski (2015) realizaram estudo para avaliar a evolução do custo, com base no modelo potencial, para quatro grandes obras de pontes na Tailândia apontando as dificuldades da realização de previsões confiáveis. Jarkas (2016) desenvolveu uma metodologia para correção de indicadores de desempenho numa obra de construção no Kuwait, visando a monitoração e controle de projetos. Neste artigo, os autores adotaram o gráfico da média acumulada e o modelo potencial. Srour, Kiomjian e Srour (2016) desenvolveram um modelo que denominaram "Modelo Recursivo", a partir do potencial, que considera o aprendizado de obras passadas, os processos de esquecimento e mecanização da produção. Os autores consideram o gráfico baseado em dados unitários, o que permite uma melhor análise da evolução da aprendizagem. Panas e Pantouvakis (2017) desenvolveram uma metodologia para avaliar as variações da produtividade em projetos de construção devido a aprendizagem, adotando a análise de sensibilidade, a curva potencial e a representação logarítmica. Srour, Kiomjiam, e Srour (2018) desenvolveram uma metodologia para selecionar, a curva de melhor ajuste, entre os modelos potencial, exponencial, hiperbólico e recursivo. Os autores concluem que para projetos de longo prazo, a curva 
exponencial e o gráfico com base na média acumulada são os mais adequados e para os de curto prazo, o modelo recursivo e o gráfico com base nos dados unitários. Ralli et al. (2020) realizaram estudo comparativo entre os modelos Potencial, Stanford "B", Cúbico; Piecewise ou Stepwise e Exponencial na fabricação de caixões de concreto marítimos. Neste estudo, os autores concluíram que o modelo cúbico apresentou melhor desempenho, para dados unitários e cumulativos ao se utilizar dados históricos. Na previsão de desempenho futuro, o modelo Stanford "B" apresentou os melhores resultados para os dados unitários e o potencial para dados cumulativos. Entretanto, os autores ressaltam que o modelo potencial produziu resultados aceitáveis para todos os cenários examinados.

No que diz respeito à soldagem, Cadaval Júnior et al. (2013) avaliaram entre os modelos Potencial, Exponencial, Hiperbólico e Logarítmico, aquele que melhor representa a aprendizagem de soldadores na indústria da construção naval, concluindo que a curva exponencial apresentou o melhor ajuste. Ammar e Samy (2015) avaliaram, entre doze combinações de modelos de curvas de aprendizagem e representações gráficas, qual a mais adequada para representar a aprendizagem na soldagem, em obras de gasodutos no Egito. Segundo os autores, quanto ao ajuste, o modelo cúbico apresentou os melhores resultados e o potencial o pior, seguido pelo exponencial. Entretanto, os autores consideram as curvas logarítmicas, elaboradas com base na média acumulada, as melhores formas de representação.

Pellegrino, Constantino, Pietroforte, e Sancillo (2012), realizaram estudo com foco na indústria de construção de prédios com vários andares na Itália, utilizando o modelo potencial, no qual detectaram ganhos de produtividade devido a repetição das tarefas. Os autores mencionam que os efeitos da aprendizagem na melhoria da produtividade podem ser perdidos, caso as condições do canteiro de obras se modifiquem. Stroieke, Anzanello, e Sanson (2013) desenvolveram uma rotina que visa integrar curvas de aprendizado e sistemáticas de clusterização, com foco na indústria de calçados brasileira, que considera a inserção de trabalhadores com perfis distintos de desempenho em uma mesma linha ou célula de produção. Raman e Varghese (2016), realizaram um Survey, na indústria da construção de edifícios na Índia concluindo que a aprendizagem de um indivíduo ou de uma equipe, devido a repetição sistemática de uma tarefa, é atribuída aos seguintes pontos:(1) maior conhecimento sobre a tarefa que está sendo executada; (2) maior familiaridade com a tarefa; (3) melhor organização do trabalho; (4) melhor coordenação; e (5) uso mais eficaz de ferramentas e métodos. Pellegrino e Constantino (2018) concluíram que os maiores ganhos de produtividade verificados em uma equipe em comparação a um indivíduo, devido à 
aprendizagem, podem ser atribuídos às ações de gerenciamento e ou à aclimatação dos trabalhadores às condições existentes num canteiro.

\section{Metodologia}

As Equações 1 e 2 representam, respectivamente, os modelos Potencial e Exponencial estudados neste artigo (Anzanello \& Fogliatto, 2007).

$y(x)=C_{1} x^{b}$

Onde, y = tempo necessário para execução de uma repetição da operação em análise; $\mathrm{C}_{1}=$ tempo de execução da primeira repetição; $\mathrm{b}=$ taxa de aprendizado, variando de 0 a 1 .

$$
y(x)=C_{1} x^{b} e^{c x}
$$

Onde, y = tempo necessário para execução de uma repetição da operação em análise; $\mathrm{C}_{1}=$ melhor tempo de execução no intervalo de tempo considerado; $b=$ taxa de aprendizado, variando de 0 a $1 ; \mathrm{c}=$ constante que influi sobre a taxa de aprendizagem, variando de 0 a 1.

A amostra é constituída por dados de produtividade de soldadores com o processo TIG de tubulações de aço carbono, em obras de plataformas de petróleo da empresa A. A produtividade é expressa em cm³/Hh (Ney, 2017; Lobato, 2016; Gioia, 2015; Tabim, 2013; PROMINP, 2010; PROMINP, 2009). Na experiência da indústria brasileira, o desempenho de soldadores atinge um patamar de normalidade a partir de 10 dias do início de suas atividades (Martins, 2011; PROMINP, 2009). Considerando que os soldadores foram admitidos em datas aleatórias e soldaram em dias diferentes, os dados de produtividade coletados foram alinhados na linha do tempo da seguinte forma: o primeiro dia de solda de cada soldador foi agrupado como "DIA 1", o segundo dia como "DIA 2" e assim por diante. Visando uniformizar as condições de execução, optou-se pelo agrupamento de dados, considerando o grau de dificuldade para soldagem das juntas, adotando-se os requisitos do código ASME IX para qualificação de soldadores. Estes requisitos são resumidos no item QW 452.3 do código ASME seção IX conforme Tabela 1.

Tabela 1. Agrupamento por diâmetro do teste de qualificação de soldadores e as faixas de diâmetros qualificadas para soldagem para juntas de topo de tubos chanfradas. 


\begin{tabular}{|c|c|c|}
\hline \multirow{2}{*}{$\begin{array}{l}\text { Diâmetro do Corpo de Prova do Teste de } \\
\text { Qualificação do Soldador } \\
\text { Pol. (mm) }\end{array}$} & \multicolumn{2}{|c|}{$\begin{array}{l}\text { Diâmetro Externos Qualificados } \\
\text { Pol. (mm) }\end{array}$} \\
\hline & Mínimo & Máximo \\
\hline Menor que 1" $(25 \mathrm{~mm})$ & $\begin{array}{l}\text { Tamanho do corpo de prova } \\
\text { soldado no teste de } \\
\text { qualificação }\end{array}$ & Ilimitado \\
\hline Entre 1"(25 mm) a $27 / 8 "(73 \mathrm{~mm})$ & $1 ”(25 \mathrm{~mm})$ & Ilimitado \\
\hline Maior do que $27 / 8 "$ (73 mm) & $27 / 8 "(73 \mathrm{~mm})$ & Ilimitado \\
\hline
\end{tabular}

Fonte: Item QW - 452.3 - ASME SEÇÃO IX - (ASME, 2010).

Organizou-se os dados de produtividade num intervalo de 10 a 20 dias de desempenho a partir do "DIA 1', em dois conjuntos: "Produtividade Média" (PM) e "Produtividade Ideal" (PI). A "Produtividade Média" corresponde à média das produtividades dos soldadores por dia conforme Equação 3.

$P M=\sum_{i=n}^{x}\left(\frac{t c m^{3}{ }_{s n}}{t H h_{s n}}+\frac{t c m^{3}{ } n+1}{t H h_{s n+1}}+\frac{t c m^{3}{ }_{s x}}{t H h_{s x}}\right) / n$

Onde:

PM = Produtividade Média diária

tcm $=$ Total de $\mathrm{cm}^{3}$ depositados

$\mathrm{tHh}=$ Total de homem-horas utilizados

$n=\mathrm{n}^{\mathrm{o}}$ total de soldadores

No caso da "Produtividade Ideal", o cálculo é adaptado do trabalho realizado por Shehata e El-Gohary (2011), onde são escolhidas as cinco maiores produtividades diárias e destes valores é extraída a mediana.

$\mathrm{Na}$ modelagem das curvas potencial e exponencial expressas nas Equações 1 e 2, ajustam-se modelos não lineares aos dados empíricos, através de estimação heurística de parâmetros (Cadaval Júnior et al., 2013). Os parâmetros dos modelos são ajustados pelo método dos mínimos quadrados, com a utilização da ferramenta Solver do Software Excel, visando minimizar a soma dos quadrados dos resíduos (Esteves, 2008). Antes da realização dos ajustes para os dois modelos, se adotou como parâmetros iniciais os seguintes valores: curva potencial - constante $\mathrm{C}_{1}$ - média da produtividade dos soldadores do "DIA 1" e o parâmetro b - 0,5; curva exponencial - constante $\mathrm{C}_{1}$ - melhor desempenho no intervalo de tempo 
considerado, parâmetro b - 0,5 e o parâmetro c - 0,5 (Anzanello, 2004).

Para avaliação da qualidade do ajuste utilizou-se os seguintes métodos: soma dos quadrados dos resíduos, coeficiente de correlação (rs) de Spearman e método gráfico. O primeiro foi escolhido como sugere Anzanello (2004), onde o modelo que apresenta o menor valor da soma dos quadrados dos resíduos é o de melhor ajuste. Na determinação da soma dos quadrados dos resíduos, o resíduo ei é calculado pela diferença entre os dados observados e estimados, conforme Equação 4 (Anzanello, 2004).

$e_{i}=y_{i}-\hat{y}_{i}$

Onde,

$e_{i}=$ i-ésima observação;

$y_{i}=$ Valores observados;

$\hat{y}_{i}=$ Valores estimados.

A correlação (rs) visa medir a relação entre duas variáveis em uma escala ordinal de medição se o tamanho da amostra é $n \geq 4$ (Corder \& Foreman, 2011). Segundo Stevenson (2001), o coeficiente de correlação (rs), cujo cálculo é apresentado na Equação 5, indica o grau de alinhamento entre as variáveis. O coeficiente de correlação (rs) pode variar de -1.00 a +1.00 , a saber:

Coeficiente de correlação para uma relação direta $-\mathrm{rs}=0.0$ - Força da Relação Nenhuma; $0.0<$ rs $\leq 0.1$ - Força da Relação - Fraca: $0.1<$ rs $<0.5$ - Força da Relação Moderada: $0.5 \leq$ rs $<1$ - Força da Relação - Forte: rs =1.0 - Força da Relação - Perfeita:

Coeficiente de correlação para uma relação indireta - rs = 0.0 - Força da Relação Nenhuma; $00.0>$ rs $\geq$-0.1- Força da Relação - Fraca: $0.1<$ rs $<0.5$ - Força da Relação Moderada: $-0.1>$ rs > -0. 5- Força da Relação - Forte: rs = - 1.0 - Força da Relação Perfeita.

$r_{s}=1-\frac{6 \sum D_{i}^{2}}{n\left(n^{2}-1\right)}$

Onde, $\Sigma \mathrm{D}^{2}=$ soma dos quadrados das diferenças entre os postos ocupados pelas funções sob análise; $\mathrm{n}=$ número de observações.

O método gráfico consiste em avaliar, visualmente, o grau de aderência entre as curvas de aprendizado, antes e após a regressão não linear, verificando qual delas representa melhor o 
conjunto de dados de campo (Stevenson, 2001; Esteves (2008).

No ajuste das curvas, se utilizou a "Produtividade Média" e a "Produtividade Ideal", que representam dados de produtividade de diâmetros diferentes, que correspondem a graus de dificuldades, aproximadamente semelhantes, segundo o ASME SEÇÃO IX, porém, não iguais. Assim, buscou-se avaliar se a composição da amostra influencia na qualidade do ajuste. No caso da "Produtividade Ideal" se avaliou a influência da "Amplitude" e da "Produtividade Média" a do "Coeficiente de Variação". Neste sentido, se avaliou se a variação do coeficiente de variação tem influência nos valores da soma do quadro dos resíduos, resultante do ajuste das curvas aos modelos estudados. Segundo Mohallem, Tavares, Silva, e Freitas (2008), o Coeficiente de Variação (CV)" é uma medida de dispersão determinada pela razão entre o desvio padrão $(\sigma)$ e a média $(\bar{x})$, conforme Equação 6.

$C V=\sigma / x$

A Equação 7 apresenta o cálculo da “Amplitude" segundo Piana, Machado, e Selau (2009), que corresponde à diferença entre valores máximo (ES) e mínimo (EI), entre os valores selecionados para determinação da "Produtividade Ideal".

Amplitude $=E S-E I$

\section{Resultados e Discussão}

4.1 - Comparação entre os comportamentos da Produtividade Média e da Produtividade Ideal com base nos dados de campo

Os valores 'das médias da "Produtividade Média (PM)", da "Produtividade Ideal (PI)" e do “Coeficiente de Variação (CV)", e da "Amplitude" determinados com base nos dados de campo, são os seguintes:

Diâmetros entre 1 a 2 7/8" - PM =2,19 cm³/Hh; Coe = 0,29, PI = 2,98 cm³/Hh, Amplitude $=1,94 \mathrm{~cm}^{3} / \mathrm{Hh}$;

Diâmetros maiores do que $27 / 8 "-\mathrm{PM}=4,71 \mathrm{~cm}^{3} / \mathrm{Hh} ; \mathrm{CV}=0,44, \mathrm{PI}=8,61 \mathrm{~cm}^{3} / \mathrm{Hh}$, Amplitude $=2,67 \mathrm{~cm}^{3} / \mathrm{Hh}$.

Os gráficos da Figura 1 descrevem o comportamento dos dados de campo da "Produtividade Média" e da "Produtividade Ideal", para as duas faixas de diâmetro. 
Figura 1. Comportamento da "Produtividade Média" (PM) e da "Produtividade Ideal" (PI) determinadas a partir dos dados de campo para diâmetros entre 1" a $27 / 8$ " e maiores que 2 $7 / 8 ”$.
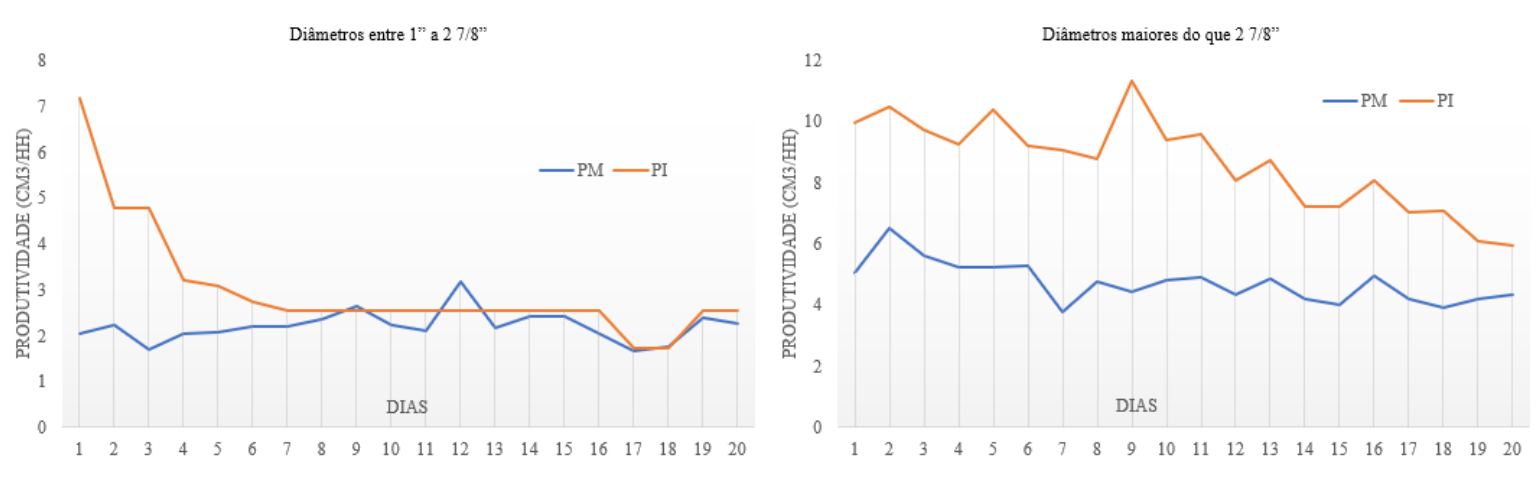

Fonte: Autores (2020).

Esperava-se que a média da "Produtividade Média" para os diâmetros maiores do que 2 7/8" fosse superior, o que foi confirmado, pois, sabe-se que quanto maior o diâmetro, melhor é o desempenho dos soldadores, tendo em vista que o grau de dificuldade é menor. As médias do "Coeficiente de Variação", para as duas faixas de diâmetro, são elevadas, o que pode influenciar na qualidade do ajuste. O "Coeficiente de Variação" é um indicador da variabilidade da produtividade global, que envolve as atividades do processo de soldagem e da ociosidade. Os eventos geradores da ociosidade, possuem variabilidade, significativamente, maior do que as atividades do processo de soldagem e tendem a aumentar os valores do "Coeficiente de Variação" (Gioia, 2015; Ney,2016).

A média da "Produtividade Ideal" é superior à da "Produtividade Média", para as duas faixas de diâmetro, o que era esperado, pois, a "Produtividade Ideal" reproduz as condições ideais de execução em que o impacto da improdutividade é reduzido (Shehata \& El-Gohary, 2011; Lobato, 2015; Tabim, 2013). Entretanto, a relação entre a "Amplitude" e a "Produtividade Ideal" média foram, respectivamente, 0,65 para a faixa de diâmetros entre 1" e 2 7/8" e 0,31 para diâmetros $27 / 8$ ”, o que poderá influir na qualidade do ajuste, sobretudo, para a relação de maior valor. A relação entre as médias da "Produtividade Média" e da "Produtividade Ideal" para as duas faixas de diâmetros são: 0,73 para diâmetros entre 1" e 2 7/8" e 0,54 para 


\begin{tabular}{c|c|c|c|c} 
Potencial & $\mathbf{1}$ a 2 7/8" & 2,04 & 0,03 & \\
\hline Potencial & Maiores do que 2 7/8" & 5,82 & $-0,06$ & \\
\hline Exponencial & $\mathbf{1}$ a 2 7/8" & 1,88 & 0,16 & $-0,02$ \\
\hline Exponencial & Maiores do que 2 7/8" & 5,49 & 0,05 & $-0,02$ \\
\hline
\end{tabular}

Fonte: Autores (2020).

Observando-se a Tabela 2, para o modelo potencial entre 1" a 2 7/8", se verifica que o valor do parâmetro $\mathrm{C}_{1}$, após ajuste, foi de 2,04 e antes do ajuste 2,03. Para diâmetros maiores do que 2 7/8", o valor de $C_{1}$ após ajuste foi de 5,82 e antes do ajuste, 5,03. Nos dois casos, o valor de $\mathrm{C}_{1}$, antes do ajuste, corresponde do "Produtividade Média" do "DIA 1" (Anzanello, 2004). Considerando o ajuste ao modelo exponencial, para diâmetros entre 1" e 2 7/8", o valor de $\mathrm{C}_{1}$, após ajuste, é de 1,88 e o estimado antes do ajuste, foi de 3,15 , obtido no "DIA 12". Para diâmetros maiores do que $27 / 8$ ", o valor de $\mathrm{C}_{1}$ antes do ajuste foi de 6,47 , obtido no “DIA 2", e após ajuste, 5,49. Nas duas faixas de diâmetro, o valor de $\mathrm{C}_{1}$ antes do ajuste, foi determinado adotando-se o critério do melhor desempenho no intervalo de tempo considerado (Anzanello, 2004). Cabe destacar, que os valores de $\mathrm{C}_{1}$ obtidos após ajuste aos dois modelos, para as duas faixas de diâmetro, foram determinados visando a obtenção da menor soma do total do quadrado dos resíduos (Srour et al., 2018).

Nos modelos potencial e exponencial um valor de b positivo, significa a ocorrência de aumento de aprendizado (Anzanello \& Fogliatto, 2007). Na Tabela 2 se verifica que os valores de b, para os dois modelos, são positivos, exceto para o potencial e diâmetros maiores do que $27 / 8$ ". Estes resultados estão alinhados com o artigo de Cadaval Júnior et al. (2013), o que não ocorre para b negativo, para este tipo de curva. Observa-se que os valores de b, positivos ou negativos, são de baixa magnitude, significando que o aumento ou redução da aprendizagem são pouco expressivos.

Segundo a Tabela 2, o parâmetro c do modelo exponencial, para as duas faixas de diâmetros, apresenta valores negativos, indicando a existência de um fator que reduz o efeito do aprendizado (Anzanello \& Fogliatto, 2007), o que está em desacordo com (Cadaval Júnior et $a l ., 2013)$. A magnitude de c é baixa, o que significa que o efeito na redução do aprendizado é reduzido.

Figura 2. Comportamento da curva de aprendizado potencial e exponencial aplicada a “Produtividade Média" (PM) nas faixas de diâmetros entre 1" a 2 7/8" e maior que 2 7/8". 

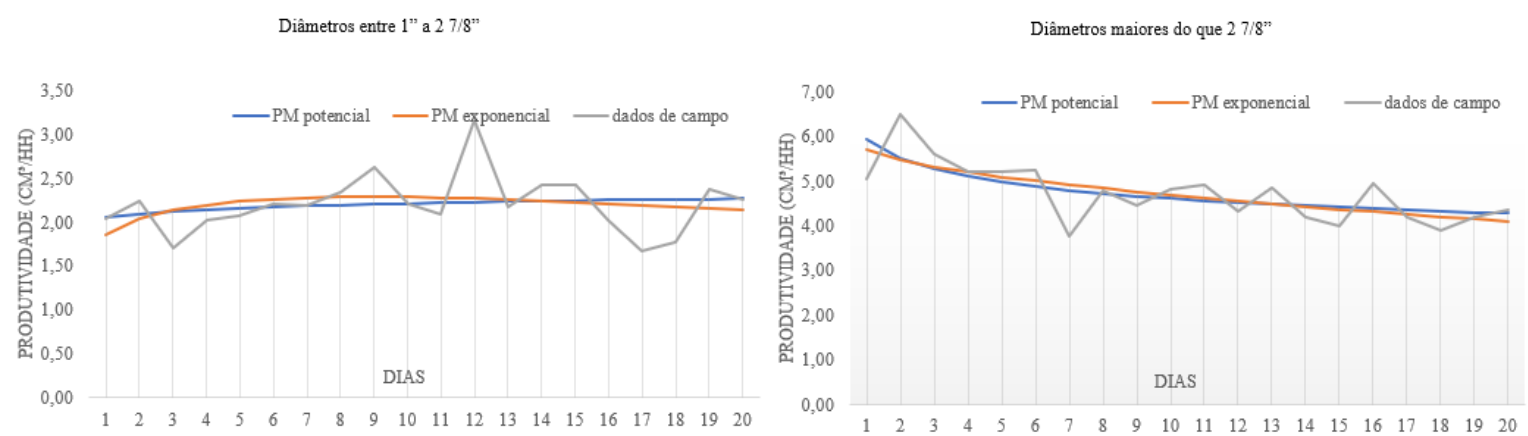

Fonte: Autores 2020.

Analisando-se o comportamento dos parâmetros b, para o modelo potencial, e b e c, para o exponencial, para as curvas ajustadas com base na "Produtividade Média", se verifica que ocorreu um aumento insignificante do aprendizado ou uma leve redução do mesmo. Esta tendência é corroborada pelos gráficos da Figura 2. PROMINP (2009), PROMINP (2010) e Martins (2011), mencionam que nos canteiros de obra do Brasil, na construção de plantas da indústria de petróleo e gás natural, os soldadores atingem seu potencial de produção máxima ao fim de 10 dias, em decorrência da acomodação às condições do canteiro. Embora, o trabalho de Lee et al. (2015) trate de trabalhadores que atuam na construção de edifícios, as semelhanças de abordagem das condições de execução são semelhantes àquelas verificadas na construção de plantas industriais no Brasil (PROMINP, 2009; PROMINP; 2010, Martins, 2011). Assim, esperava-se, ao menos, que houvesse um leve aumento do aprendizado. Entretanto, o comportamento dos parâmetros b e c e a observação gráfica da Figura 2, evidenciam que, isto só se verifica para diâmetros entre 1" a 2 7/8".

A Figura 2 possibilita avaliar a qualidade do ajuste e o modelo de melhor aderência, pelo método gráfico, para os modelos potencial e exponencial ajustados aos dados da "Produtividade Média", para as duas faixas de diâmetros (Stevenson, 2001; Esteves, 2008).

Observando-se a Figura 2, verifica-se que, graficamente, as curvas ajustadas aos dois modelos são muito próximas, dificultando a seleção daquele que apresenta as melhores condições de ajuste.

$\mathrm{Na}$ Tabela 3 são apresentados os valores da soma dos quadrados dos resíduos e da correlação (rs) resultante do ajuste dos dados reais da "Produtividade Média" aos modelos potencial e exponencial, para as faixas de diâmetro de 1" a $27 / 8$ " e maiores do que 2 7/8". Os resultados demonstram que para diâmetros entre 1 a 2 7/8" o modelo exponencial apresenta a menor soma dos quadrados dos resíduos e o melhor ajuste. No caso dos diâmetros maiores do que 2 
7/8", o potencial apresenta condições de ajuste levemente superiores (Anzanello, 2004). Quanto a correlação (rs), a faixa de diâmetros entre 1 a 2 7/8", registra a maior correlação rs com os dados reais, indicando as melhores condições de ajuste. Entretanto, para diâmetros maiores do que $27 / 8$ ” os valores de (rs) são iguais (Corder \& Foreman, 2011).

Tabela 3. Comparação entre os critérios de ajuste, soma dos quadrados dos resíduos (SQR) e correlação (rs) determinados para os modelos de curva potencial e exponencial com base na "Produtividade Média".

\begin{tabular}{c|c|c|c|c}
\hline \multirow{2}{*}{ Diâmetros } & \multicolumn{2}{|c|}{ 1" a 2 7/8" } & \multicolumn{2}{c}{ Maiores do que 2 7/8" } \\
\cline { 2 - 5 } & \multicolumn{3}{|c}{ Modelos das Curvas de Aprendizado } \\
\hline Critérios de Ajuste das Curvas & Potencial & Exponencial & Potencial & Exponencial \\
\hline SQR & 2,03 & 1,90 & 4,33 & 4,09 \\
\hline rs & 0,17 & 0,41 & 0,68 & 0,68 \\
\hline
\end{tabular}

Fonte: Autores (2020).

Concluindo a análise do comportamento dos modelos potencial e exponencial, considerandose os três critérios de ajuste, se verifica que o exponencial é o que melhor representa o aprendizado (Cadaval Júnior et al., 2013). Entretanto, o modelo potencial também apresenta bons resultados (Leite, 2002; Anzanello \& Fogliatto, 2007; Ralli et al., 2020). Ammar e Samy (2015) concluíram em seu estudo sobre soldagem de gasodutos, que o modelo cúbico é o mais adequado e o exponencial apresentou melhores resultados que o potencial.

No que diz respeito a influência do "Coeficiente de Variação" na qualidade do ajuste, para a faixa de diâmetros entre 1" e $27 / 8$ ", conforme mencionado na seção 4.1, a média dos valores do "Coeficiente de Variação" para a "Produtividade Média", foi de 0,29 e os valores da soma dos quadrados dos resíduos foram, respectivamente, 2,03 para o modelo potencial e 1,90 para o exponencial. Para diâmetros maiores do que $27 / 8$ ", a média do "Coeficiente de Variação" foi de 0,44 e os valores da soma dos quadrados dos resíduos foram de 4,33 para o modelo potencial e 4,09 para o exponencial. Assim, os resultados indicam que a variação do "Coeficiente de Variação" influenciou a qualidade do ajuste nos dois modelos. Neste sentido, o agrupamento de dados de produtividade de acordo com os requisitos do ASME SEÇÃO IX (2010), mostrou-se adequado para as duas faixas de diâmetros. Por outro lado, os resultados indicam que a qualidade do ajuste poderia ser melhorada se a "Produtividade Média" fosse determinada para cada diâmetro de tubulação, entretanto, esta medida é mais trabalhosa e 
O parâmetro c apresenta um valor positivo, conforme Tabela 4, para diâmetros entre 1" a 2 7/8", indicando a existência de um fator que aumenta o aprendizado (Anzanello \& Fogliatto, 2007). Estes resultados estão alinhados com Cadaval Júnior et al. (2013), entretanto, para diâmetros maiores do que 2 7/8”, c assume um valor negativo. Os valores de b e c, positivos ou negativos, são de baixa magnitude, sendo que, tanto no caso do parâmetro b no modelo potencial, quanto para a combinação de b e c no exponencial, se observa um efeito de redução da aprendizagem, conforme se observa na Figura 3.

Tabela 4. Comportamento dos parâmetros do modelo potencial e exponencial para faixas de diâmetro de 1 a 2 7/8" e maiores que 2 7/8" - Ajuste aos dados da "Produtividade Ideal".

\begin{tabular}{c|c|c|c|c}
\hline Modelo de Curva & Faixa de Diâmetros & Parâmetro C & b & c \\
\hline Potencial & 1" a 2 7/8" & 6,77 & $-0,42$ & \\
\hline Potencial & Maiores do que 2 7/8" & 10,72 & $-0,11$ & $-0,03$ \\
\hline Exponencial & $\mathbf{1 " ~ a ~ 2 ~ 7 / 8 " ~}$ & 6,96 & $-0,60$ & $-0,03$ \\
\hline Exponencial & Maiores do que 2 7/8" & 9,88 & 0,06 & \\
\hline
\end{tabular}

Fonte: Autores (2020).

Analisando-se os gráficos da Figura 3 ocorrem em todos os casos uma diminuição da aprendizagem que é mais acentuada para as curvas ajustadas ao modelo potencial para diâmetros entre 1 a 2 7/8”. Para os diâmetros maiores do que 2 7/8”, observa-se um ligeiro aumento da aprendizagem entre o "DIA 2" e o "DIA 5". Este comportamento, pode ser resultado do efeito da ocorrência do valor positivo do parâmetro c, que atua aumentando a aprendizagem, bem como, reduzindo a ação da presença do parâmetro b, que é negativo e influi no sentido inverso, considerando ainda que os dois parâmetros possuem a mesma ordem de grandeza. A determinação da "Produtividade Ideal”, segundo Shehata e El-Gohary (2011), reduz o impacto dos eventos geradores da ociosidade, na determinação da produtividade global e tendem a representar a produtividade do processo. Assim, ao contrário do que se esperava, observa-se a redução da aprendizagem ao longo tempo. Neste artigo não existem elementos que permitam a explicação deste resultado. Neste sentido, para que seja possível uma avaliação mais precisa deste comportamento é necessária uma análise detalhada do procedimento de soldagem utilizado, o que não é escopo deste trabalho.

Observando-se a Tabela 5, se verifica que a faixa de diâmetros entre 1" a 2 7/8" apresenta os menores valores da soma dos quadrados dos resíduos e, consequentemente, as melhores condições de ajuste para os dois modelos (Anzanello, 2004). A mesma tendência se repete 
para a correlação (rs) (Corder \& Foreman, 2011). Nesta faixa de diâmetros os valores da correlação (rs) e da soma dos quadrados dos resíduos são muito próximos para os dois modelos. O modelo exponencial registra valores, ligeiramente, menores da soma dos quadrados dos resíduos e maiores da correlação (rs), indicando melhores condições de ajuste (Cadaval Júnior et al., 2013). Quanto à representação gráfica, o comportamento dos dois modelos também é bem próximo (Stevenson, 2001; Esteves, 2008). Considerando os três requisitos, o modelo exponencial é o que melhor representa o aprendizado. Entretanto, o modelo potencial também oferece boas condições de ajuste (Everett e Farghal, 1997; Leite, 2002; Thomas, 2009; Ralli et al., 2020).

Figura 3. Comportamento da curva de aprendizado potencial e exponencial aplicada a "Produtividade Ideal" nas faixas de diâmetros de 1" a 2 7/8" e maior que 2 7/8"
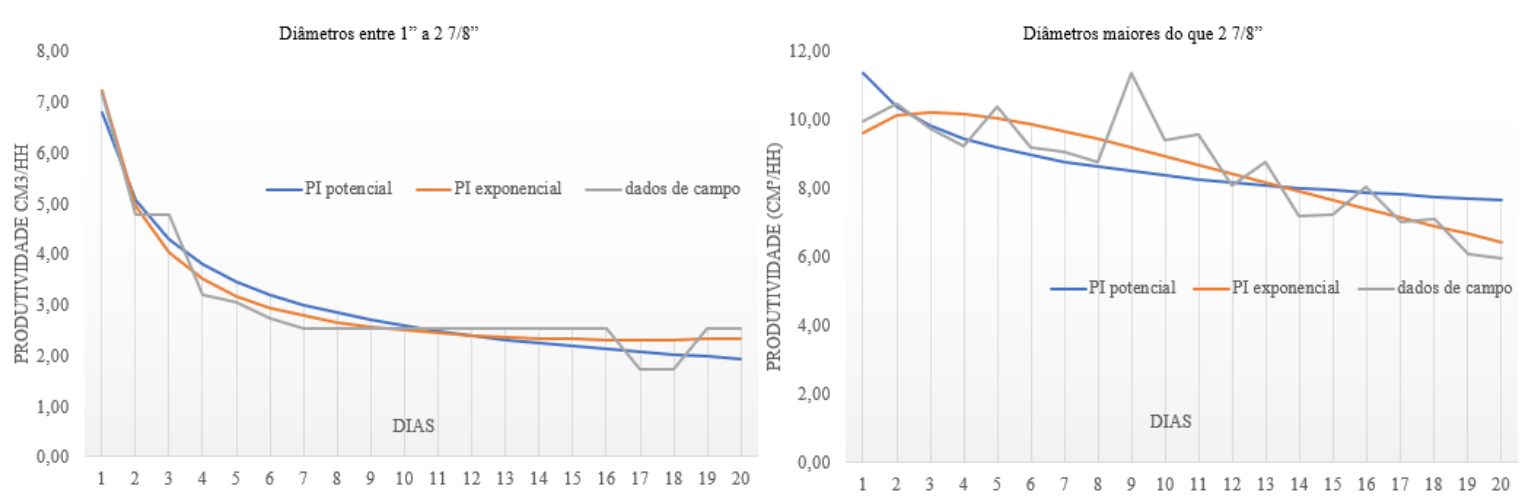

Fonte: Autores (2020)

Para diâmetros maiores do que $27 / 8$ ", os valores da correlação (rs) indicam a existência de uma correlação forte para os dois modelos. Entretanto, o valor de (rs) para o potencial é ligeiramente superior, apresentando melhores condições de ajuste (Corder \& Foreman, 2011). Por outro lado, considerando o critério de menor valor da soma dos quadrados dos resíduos, o valor obtido para o modelo potencial é mais que o dobro do exponencial e apresenta as melhores condições de ajuste (Anzanello, 2004). Quanto a representação gráfica, como se observa na Figura 3, o ajuste aos dois modelos é bem próximo. Considerando-se o atendimento aos três requisitos, se verifica que em dois deles, as condições de ajuste aos dois modelos são semelhantes. Entretanto, quando se trata do menor valor da soma dos quadrados dos resíduos, a diferença entre os dois modelos é bem significativa, sendo que o exponencial 
apresenta as melhores condições de ajuste.

Tabela 5. Comparação entre os critérios de ajuste determinados para os modelos de curva potencial e exponencial com base na "Produtividade Ideal".

\begin{tabular}{|c|c|c|c|c|}
\hline \multirow{2}{*}{ Diâmetros } & \multicolumn{2}{|l|}{ 1" a 2 7/8" } & \multicolumn{2}{|c|}{ Maiores do que 2 7/8" } \\
\hline & \multicolumn{4}{|c|}{ Modelos das Curvas de Aprendizado } \\
\hline Critérios de Ajuste das Curvas & Potencial & Exponencial & Potencial & Exponencial \\
\hline SQR & 2,93 & 1,84 & 22,56 & 10,57 \\
\hline rs & 0,98 & 0,99 & 0,87 & 0,83 \\
\hline
\end{tabular}

Fonte: Autores (2020).

A partir dos dados registrados na seção 4.1 se verifica que a relação entre as médias da "Amplitude" e da "Produtividade Ideal" é de 0,65 para diâmetros de 1" a 2 7/8" e de 0,31 para diâmetros maiores do que 2 7/8". A Tabela 5 mostra que os valores da soma dos quadrados dos resíduos, para diâmetros entre 1" e 2 7/8" são, significativamente, inferiores aos obtidos para diâmetros maiores do que 2 7/8". Estes resultados não permitem concluir pela influência da "Amplitude" na qualidade do ajuste.

\section{4 - Efeito da Aprendizagem na Produtividade}

A utilização dos dados da "Produtividade Média" resultou em melhores condições de ajuste do que para a "Produtividade Ideal", sobretudo, para diâmetros maiores do que 2 7/8". Assim, no que tange a indústria brasileira, a utilização da "Produtividade Média" como indicador de produtividade para previsões e correções de prazo durante a realização de um empreendimento, parece ser mais adequada.

Tendo em vista que a "Produtividade Média" apresentou as melhores condições de ajuste, se avaliou o efeito da aprendizagem com base neste indicador. Assim, se observou, nas duas faixas de diâmetro, que não se houve melhoria da "Produtividade Média", o que está em desacordo com a literatura, tanto para a soldagem, quanto para outras atividades da indústria da construção (Ammar \& Samy, 2015; Anzanello, 2004; Cadaval Júnior et al., 2013; (Pellegrino et al., 2012; Jarkas, 2016; (Pellegrino \& Constantino, 2018; Raman \& Varghese, 2016; Srour et al., 2016; Ralli et al., 2020). A melhoria da produtividade a partir do $10^{\circ}$ dia não se verificou (Martins, 2011; PROMINP, 2009). Esperava-se que esta melhora viesse a ocorrer, ao menos, em função da aclimatação dos trabalhadores às condições do local de 
trabalho (Pellegrino \& Constantino, 2018; Ralli et al., 2020). Cabe destacar, que a soldagem das juntas de tubulações são atividades individuais, dependem da habilidade de cada soldador e a amostra estudada considera a média diária dos soldadores e não produto de um trabalho de equipe. Assim, conforme preconizado por Pellegrino e Constantino (2018) não se observam as condições para que haja melhoria da produtividade em função do trabalho de equipe. Por outro lado, os eventuais efeitos decorrentes da heterogeneidade da composição da equipe sobre a performance da produção, também não se aplicam (Stroieke, Anzanello, \& Sanson, 2013).

\section{Conclusões e Sugestões para Trabalhos Futuros}

Os resultados demonstraram que as curvas elaboradas a partir da produtividade média representam de forma mais adequada a aprendizagem do que as construídas com base na produtividade ideal.

O modelo exponencial apresentou condições de ajuste ligeiramente superiores ao potencial. Neste sentido, o potencial pode ser utilizado obtendo resultados aceitáveis, o que é mencionado na literatura.

Quanto a composição da amostra, os resultados obtidos a partir da produtividade média para os modelos potencial e exponencial, resultaram ajustes de qualidade razoável, para as faixas de diâmetro de 1" a 2 7/8" e maiores do que 2 7/8". Assim, o estudo do comportamento do aprendizado, agrupando os diâmetros, segundo o grau de dificuldade, nas faixas estabelecidas pelo código ASME IX mostrou-se viável.

Quanto a aprendizagem, não se verificou ganhos de produtividade no intervalo de tempo estudado, o que não era esperado, tanto devido a melhoria do processo, quanto pela aclimatação dos soldadores às condições do local de trabalho. No que diz respeito a utilização de um indicador da produtividade, para estimativas e correções de prazo, durante um empreendimento, a adoção da produtividade média a partir do $10^{\circ}$ dia, conforme praticado pela indústria brasileira, parece ser razoável.

Neste artigo, comparou-se o comportamento de dois modelos de curvas de aprendizagem. Assim, sugere-se que em trabalhos futuros se avalie, comparativamente, o comportamento de outros modelos. 
Neste trabalho, utilizou-se dados unitários e o foco do estudo situou-se num curto intervalo de tempo. Assim, como se observa na literatura, trabalhos que utilizem dados cumulativos e que envolvam prazos de execução mais longos, podem ser desenvolvidos.

\section{Referências}

Adrian, J. J. (2004). Construction Productivity: Measurement and improvement. Champagne. IL: Stipes Publishing.

Ammar, M., \& Samy, M. (2015). Learning curve modelling of gas pipeline construction in Egypt. International Journal of Construction Management, 15, pp. 229-238.

Anzanello, M. (2004). Curvas de aprendizado como balizadoras da alocação de modelos de produtos a equipe de trabalhadores. (Dissertação Mestrado). Universidade Federal do Rio Grande do Sul, Rio Grande do Sul.

Anzanello, M., \& Fogliatto, F. (2007). Curvas de aprendizado: estudo da arte e perspectivas de pesquisa. Gestão \& Produção, 1, pp. 109 - 123.

Arditi, D., \& Mochtar, K. (2000). Trends in productivity improvement in the U.S. construction industry. Construction Management Economics, 18, pp. 15 - 27.

ASME - American Society of Mechanical Engineers. (2010). ASMEIX - Qualification Standard for Welding and Brazing Procedures, Welders, Brazers, and Welding and Brazing Operators. New York, New York, USA: American Society of Mechanical Engineers .

AWS - American Welding Society. (2002). Welding - Related Expenditures and Productivity Measurement in U.S. Manufacturing, Construction, and Mining Industries. American Welding Society.

Brockmann, C., \& Brezinski, H. (2015). Experience curve effects in bridge construction. Procedia Economics and Finance, 21, pp. $563-570$.

Cadaval Júnior, D., Duarte, E., \& Paes, R. (2013). Curvas de aprendizado aplicadas a análise da produtividade de soldadores. Vetor, 23, pp. 29-38.

Carpinetti, L. C. (2010). Gestão da qualidade conceitos e técnicas (1 ed.). São Paulo, São Paulo, Brasil: Atlas S.A.

Chia, F. C. (2014). Construction and economic development: the case of Malaysia. International Journal of Construction Management, 12, pp. 23-35.

Chia, F. C., Skitmore, M., Runeson, G., \& Bridge, A. (2012). An analysis of construction productivity in Malaysia. Construction Management and Economics, 30, pp. 1-15.

Corder, G., \& Foreman, D. (2011). Nonparametric Statistics for Non-Statisticians: A Step-by-Step Approach. John Wiley \& Sons. 
Zhang, D., Nasir, H., \& Haas, C. (2017). Development of an internal benchmarking and metrics model for industrial construction enterprises for productivity improvement. Canadian Journal of Civil Engineering, 44, pp. 518-529.

Zhang, L., Zou, X., \& Z. Kan, Z. (2014). Improved strategy for resource allocation in repetitive projects considering the learning effect. Journal of Construction Engineering and Management, 140, pp. 1 - 8. 